\title{
Consumer Knowledge of Over-the-Counter Phenazopyridine
}

Chib-Wen Shi, M.D, MSHS ${ }^{1}$

Steven M. Asch, M.D, MPH ${ }^{2,3,4}$

Eve Fielder, $\mathrm{Dr}_{\mathrm{P}} \mathrm{H}^{5}$

Lillian Gelberg, MD, MSPH ${ }^{6}$

Michael B. Nichol, $P b D^{7}$

'Department of Family \& Preventive Medicine, University of California, San Diego, San Diego, Calif

${ }^{2}$ RAND, Santa Monica, Calif

${ }^{3}$ Veteran's Affairs Greater Los Angeles Healthcare System, Los Angeles, Calif

${ }^{4}$ Department of Medicine, University of California, Los Angeles, Los Angeles, Calif

${ }^{5}$ Institute of Social Science Research, University of California, Los Angeles, Los Angeles, Calif

${ }^{6}$ Department of Family Medicine, University of California, Los Angeles, Los Angeles, Calif

${ }^{7}$ Department of Pharmaceutical Economics and Policy, University of Southern California, Los Angeles, Calif

Conflicts of interest: none reported

\section{CORRESPONDING AUTHOR}

Chih-Wen Shi, MD, MSHS

Department of Family \& Preventive Medicine

University of California, San Diego

9500 Gilman Dr, Mailcode-0807

La Jolla, CA 92093

cwshi@ucsd.edu

\begin{abstract}
BACKGROUND Effective use of over-the-counter (OTC) medications depends on purchasers' knowledge of their indications. This study examines consumer knowledge regarding the urinary tract analgesic phenazopyridine, which recently became available without prescription.
\end{abstract}

METHOD We conducted a cross-sectional survey of a stratified cluster random sample of purchasers of OTC phenazopyridine $(N=434)$ in 31 Los Angeles retail pharmacies.

RESULTS The response rate was $58 \%$. Only $29 \%$ correctly characterized the likely cause of their symptoms, and only $57 \%$ correctly characterized the action of the drug. Worse consumer knowledge was associated with nonwhite race, first-time use, and less contact with health providers.

CONCLUSION Many consumers possess poor knowledge about phenazopyridine, potentially leading to undertreatment, especially in groups with worse access to care.

Ann Fam Med 2004;2:240-244. DOI: 10.1370/afm.61.

\section{INTRODUCTION}

$\mathrm{n}$ response to demands for more consumer choice and reduced health care costs, there has been a movement to make prescription drugs available as over-the-counter (OTC) products. ${ }^{1-5}$ More than 600 OTC drugs now use ingredients and dosages that 20 years ago were available only by prescription. ${ }^{2}$ The retail market value of these reclassified products totals about $\$ 8.8$ billion and will likely continue to increase in the future. ${ }^{2}$ This abundant availability of OTC drugs allows the public to self-medicate and to treat various symptoms. Yet despite its substantial impact on self-care, little is known about whether members of the public have adequate knowledge to use these drugs correctly.

Effective self-care involves a complex sequence of tasks, including diagnosing the condition and its cause, selecting proper drug therapy, and monitoring treatment effectiveness. Whereas patients and health care providers have always shared these decisions to some extent, the current availability of OTC medications allows greater potential range of decision making for patients acting without direct provider guidance. To date, no studies of OTC drugs have examined the users' understanding of the cause of symptoms and of indications for using the drug.

To address this gap, we conducted a cross-sectional study to evaluate the knowledge of consumers purchasing a recently widely marketed OTC medication, phenazopyridine (Pyridium). Phenazopyridine is a urinary tract analgesic for adjunctive treatment of pain associated with urinary tract infections in addition to antibiotics aimed at the underlying microbial infection. ${ }^{4}$ It is available as an OTC medication for temporary relief of dysuria while the patient is awaiting medical evaluation and treatment. We hypothesized that consumers would commonly have incorrect beliefs 
about the likely infectious cause of dysuria-associated symptoms. We further hypothesized that consumers are likely to incorrectly believe that this drug has antibacterial or curative effects. In our study, we measured the rates of incorrect knowledge among purchasers of phenazopyridine in 31 pharmacies in the Los Angeles area and investigated factors that might be correlated with poor consumer knowledge.

\section{METHODS}

We conducted a cross-sectional survey of a stratified clustered random sample of purchasers of OTC phenazopyridine $(\mathrm{N}=434)$ in 31 Los Angeles pharmacies from September 2000 to February 2001.

For the selection of sites, the corporate office of a well-known, national retail pharmacy chain provided a list of their pharmacies in the Los Angeles County. From this list (79), we chose as our sampling frame a subset of 48 pharmacies located within 20-mile radius from central Los Angeles. From this subset, 4 pharmacies with convenient geographic location and good contacts were chosen with certainty as pilot sites. Because income has been shown to be associated with OTC medication use, ${ }^{7}$ we ranked the remaining 44 pharmacies by the 1989 US census per capita income reported for their respective ZIP codes. Thirty pharmacies were randomly selected from this ranked list. Because of changes in management, 2 of the 30 pharmacies sampled through this method and 1 original pilot site later declined participation; therefore, a total of 31 pharmacies served as survey sites for our main study.

Respondent weights were calculated to correct for sampling, interpharmacy site differences in nonresponse rate, and data collection duration. We estimated the design effect from weighting using standard formulas to be 1.001 . The design effect from clustering was similarly small.

At the time of purchase, consumers completed a 25 -item self-administered anonymous questionnaire that took approximately 3 minutes. This questionnaire, available in English and Spanish, was pretested before its administration for readability and comprehension using one-on-one cognitive interviews with 90 clinic patients and 30 actual phenazopyridine consumers. The Spanish questionnaire was verified with professional forward and backward translations. A copy of the questionnaire is available upon request from the corresponding author.

Because each pharmacy required detailed education regarding the research protocol, data collection began at each of the 31 pharmacies on different days over a course of 3 weeks beginning on September 24, 2000, and ended at all sites on February 16, 2001. During the study, customers were recruited by placing bilingual (English and Spanish) advertisements next to the OTC phenazopyridine products on the shelves. These advertisements directed interested customers to the pharmacy counter, where they completed the questionnaires. Participants were 18 years or older and purchased any 1 of the 3 OTC phenazopyridine products (Azo-Standard, Prodium, or Uristat) for their personal use. Those who completed a questionnaire received a $\$ 5$ discount on their purchase of the product. In an effort to adhere to a natural experiment setting for our study, the pharmacists remained as neutral consultants and were neither prohibited nor encouraged to recommend phenazopyridine to consumers. Likewise, the consumers were not cued with specific instructions to read the package labels before or during the completion of our questionnaire. The survey response rate was calculated as the number of questionnaires returned divided by the number of phenazopyridine products that were sold during the study period.

\section{Outcome Measures}

In the questionnaire, we asked the participants, "What do you think is causing your symptoms?" Respondents wrote their answers in an open-ended format. These answers were then categorized under 5 major themes determined after data collection: (1) urinary tract infection-bladder infection, (2) diet (eg, water or beverage intake), (3) personal habits (eg, bathroom use, sexual activity), (4) don't know, and (5) other environmental factors (eg, stress, unclean toilets).

We used a multiple-choice question to assess consumer's knowledge about the action of phenazopyridine. Participants were asked to check all answers that would apply to this statement: I believe this medicine will..." Potential answers included lower fever, reduce pain during urination, kill bacteria that cause infection, cure urinary tract infection, act as a water pill, and other actions, along with the opportunity to write in additional comments. We defined correct knowledge as the choice "reduce pain during urination" and incorrect knowledge as any other choices.

\section{Independent Variables}

In the development of our survey questionnaire, we applied a modified version of the Andersen behavioral model $^{8}$ to conceptualize the environmental and population (predisposing, enabling, and need) factors that affect access to medical care compared with self-care practices. Among the environmental and predisposing factors, we included advertisement, waiting for a medical appointment, friend's and family's advice, pharmacist's advice, lack of time to visit a provider, attitudes toward providers, and financial tradeoffs. Under popu- 
lation characteristics, age and gender were included as they are risk factors for urinary tract infections. We included health insurance as an enabling factor, noting that it could have different effects on consumer's behavior: insured patients might learn about phenazopyridine while visiting their provider and then purchase the product, whereas uninsured persons might choose to self-medicate. Finally, to account for need for the drug, we asked about providers' recommendation for phenazopyridine and duration of symptoms. Together, these various factors were correlated with our outcome of interest, consumer knowledge.

\section{Statistical Analysis}

Statistical analyses were performed using the Intercooled STATA 7.0 survey commands (College Station, Texas, 2001). Only 3 questionnaires had any missing data, which were imputed by mean replacement method. Zero-order correlation matrices were used to check for multicollinearity. Exploratory modeling of knowledge began with cross-tabulations. Pearson chi-square coefficients and independent sample $t$ tests were used to identify potential predictors of the outcome, knowledge of the action of phenazopyridine. We entered a total of 10 variables with an association of $P<.2$ from the bivariate analysis into a multiple logistic regression model to predict outcome of knowledge. Four variables with $P<.05$ were retained in the final models. Finally, to evaluate the goodness of fit for the final model, we applied the Hosmer-Lemeshow test on 9 distinct groups. Doing so, we obtained a Hosmer-Lemeshow chi-square with 7 degrees of freedom equal to 4.29 and $P=$.746. It appeared, therefore, that our model fit was acceptable.

\section{RESULTS}

During the study period, 746 boxes of OTC phenazopyridine were sold, and 434 consumers completed our questionnaire. The overall survey response rate was $58 \%$. As shown in Table 1, the respondents were predominantly young white women with good levels of education and income.

Although OTC phenazopyridine has been popularly advertised in the media as an interim pain reliever pending a medical appointment for a urinary tract infection, only $29 \%$ of the respondents believed their symptoms were due to a urinary tract infection or bladder infection. Forty-two percent admitted they did not know or had no ideas about what was causing their urinary tract symptoms, and another $29 \%$ attributed the cause to unlikely sources, such as diet, personal habits, or environmental factors. For example, those who attributed the cause to their diet stated that they drank

\section{Table 1. Overall Population Characteristics $(\mathrm{N}=434)$}

\begin{tabular}{|c|c|}
\hline Characteristic & $\begin{array}{c}\text { Weighted } \\
\text { Percent }\end{array}$ \\
\hline \multicolumn{2}{|l|}{ Sex } \\
\hline Female & 96 \\
\hline \multicolumn{2}{|l|}{ Ethnicity } \\
\hline White & 45 \\
\hline Nonwhite & 55 \\
\hline African American & 17 \\
\hline Asian/Pacific Islander & 11 \\
\hline Hispanic & 24 \\
\hline Other & 3 \\
\hline \multicolumn{2}{|l|}{ Education } \\
\hline Below high school & 1 \\
\hline Some high school & 5 \\
\hline High-school graduate & 23 \\
\hline Some college & 32 \\
\hline College graduate & 29 \\
\hline Postgraduate & 11 \\
\hline \multicolumn{2}{|l|}{ Annual household Income } \\
\hline$<\$ 30,000$ & 38 \\
\hline$\$ 30,00-\$ 50,000$ & 27 \\
\hline$\$ 50,001-\$ 70,000$ & 18 \\
\hline$>\$ 70,000$ & 16 \\
\hline \multicolumn{2}{|l|}{ Health insurance } \\
\hline Yes & 81 \\
\hline \multicolumn{2}{|l|}{ History of urinary tract infection } \\
\hline Yes & 63 \\
\hline \multicolumn{2}{|l|}{ Previous use of prescription phenazopyridine } \\
\hline Yes & 29 \\
\hline \multicolumn{2}{|l|}{ On concurrent antibiotic } \\
\hline Yes & 23 \\
\hline \multicolumn{2}{|c|}{ Using phenazopyridine instead of going to the doctor } \\
\hline Yes & 38 \\
\hline \multicolumn{2}{|l|}{ Beliefs about cause of urinary tract symptoms } \\
\hline Don't know & 42 \\
\hline Urinary tract infection/bladder infection & 29 \\
\hline Other & 29 \\
\hline \multicolumn{2}{|l|}{ Knowledge of drug action } \\
\hline Correct & 57 \\
\hline Incorrect & 43 \\
\hline
\end{tabular}

too much coffee, tea, soda, alcohol, or other beverages or that they did not have enough water intake. Beliefs involving personal habits included not using the bathroom often enough or unconventional sexual practices. Postulated environmental factors included stress and unclean toilets.

Although respondents had access to the package label at the time of the survey, only $57 \%$ knew phenazopyridine was a urinary tract analgesic. All other respondents thought that this drug had other actions, such as 


\section{Table 2. Factors Associated With Knowledge of Over-the-Counter Phenazopyridine}

\begin{tabular}{lcc}
\hline Factors* & Adjusted Odds Ratio (95\% CI) \\
\hline 1. White & $1.55(1.11-2.16)$ & $P$ Value \\
2. Concurrent antibiotic use & $1.91(1.10-3.3)$ \\
3. Previous use of prescription phenazopyridine & $1.95(1.07-3.5)$ \\
4. Using phenazopyridine instead of going to doctor & $0.59(0.37-0.92)$ \\
\hline $\mathrm{Cl}=$ confidence interval. & .023 \\
Note: Adjusted for the linear effects of other variables in the model. *Variables entered into the model included: sex, using phenazopyridine instead of going to doctor, pre- \\
vious use of prescription phenaopyridine, concurrent antibiotic use, race, advertisement, drug cheaper than doctor, no time to see doctor, dislike doctor, education. \\
* Variables with an adjusted odds ratio significantly different from 1.0 at the $P<.05$ level.
\end{tabular}

antibacterial or curative effects. Combining beliefs of symptom cause and knowledge of drug actions, 19\% of the phenazopyridine purchasers knew neither the cause of their symptoms nor the action of the drug, and an additional $14 \%$ poorly characterized the cause of their symptoms and also did not know the action of phenazopyridine. Furthermore, $10 \%$ believed that their symptoms were due to urinary tract or bladder infection but did not know the effects of phenazopyridine. Yet they all purchased the product.

Multiple logistic regression analyses showed that whites had 1.55 times higher odds of having correct knowledge about the drug action than nonwhites. Consumers who stated they were not using this drug instead of going to see a physician had 1.41 higher odds of having correct knowledge than those who were using this drug instead of going to see a physician. Those who were on concurrent antibiotics had 1.91 times higher odds of having correct knowledge than those not on concurrent antibiotics, and those who had previously used the prescription form of phenazopyridine had 1.95 times higher odds of having correct knowledge than those who had not used the prescription form previously.

\section{DISCUSSION}

This survey of consumers purchasing OTC phenazopyridine in 31 Los Angeles County pharmacies showed that a significant proportion had poor knowledge of the indication and mechanism of action of the drug. Nonwhite purchasers of this drug were less likely to know that phenazopyridine is a urinary tract analgesic, not a cure for infection, even controlling for previous use of this drug and for access to a physician. For phenazopyridine at least it appears that the complex chain of self-diagnosis, treatment, and monitoring needed for effective OTC use rests upon a shaky foundation. Furthermore, contacts with physicians appear to be associated with more accurate knowledge.

This study design has several unique features. First to our knowledge, it is the first OTC study in the United States that collected data directly from consumers at the time of purchase within a large number of retail pharmacies. Thus, we gained insights into exactly how the public uses an OTC medication in the realworld setting. Second, the sampling methods ensured generalizability to purchasers in a large, well-known national retail chain in an ethnically diverse geographical region.

This study has some limitations. First, in our effort to adhere to a natural experiment, the consumers were not cued with specific instructions to read the package information before responding to our questionnaire. It is conceivable that consumers read the package information after they went home and improved their knowledge in this way. We believe, however, this event occurred infrequently. Second, because the study was cross-sectional and anonymous, we do not have followup data on the respondents to determine either how they used this medication at home or the associated health outcomes. Because the consumers had to pay for this medication out-of-pocket, their decision to purchase most likely served as a proxy for use.

Third, because we could not track those consumers who elected not to participate in the study, we cannot assess for nonresponse bias. Nevertheless, our response rate of $58 \%$ represents a notable improvement in participation compared with other similar studies in community pharmacy settings. ${ }^{9}$ Fourth, our response rate denominator reflected a conservative calculation and assumed that each box was independently purchased by a different consumer. It was conceivable that any one consumer could have purchased more than 1 product or that products may have been lost or stolen during the study period, which would bias our estimate of the response rate downward.

Our study findings have striking implications for unrestricted use of phenazopyridine and perhaps for other OTC drugs as well. Presently, the Food and Drug Administration uses 3 regulatory criteria to determine whether a prescription medication should 
be available as an OTC drug. Mainly, the drug should (1) have low potential for misuse, (2) treat an easily self-diagnosed condition, and (3) contain a package label with easily understood indications, contraindications, and use instructions. ${ }^{10}$ Our study showed a surprisingly wide discrepancy between these intended guidelines and the purchasers' actual knowledge. Consumers with various urinary tract conditions probably purchased phenazopyridine simply based on its advertisement as a urinary tract pain relief product rather than based on a good understanding of the cause of symptoms or mechanism of action of the drug. For many, this lack of knowledge can potentially lead to undertreatment of urinary tract infections and other urinary tract conditions. Even though package labels contain important drug information, it appears that a more active method is necessary to diffuse knowledge effectively to the consumers. Consequently, better patient education and post-OTC marketing surveillance is essential to assure proper usage of OTC medications, particularly as popular prescription drugs, such as proton pump inhibitors and the statins, head toward the OTC marketplace. ${ }^{11,12}$

To read or post commentaries in response to this article, see it online at http://www.annfammed.org/cgi/content/full/2/3/240.

Key words: Pharmacy; survey; over-the-counter drugs; phenazopyridine

Submitted November 15, 2002; submitted, revised, April 20, 2003; accepted May 26, 2003.
Funding support: This investigation was supported by National Research Service Award F32 HS11507-01 from the Agency for Healthcare Research and Quality (AHRQ) awarded to Dr. Shi. Dr. Asch is a recipient of the Veteran's Affairs Health Services Research Development Career Development Award.

\section{References}

1. Brass EP. Changing the status of drugs from prescription to over-thecounter availability. N Engl J Med. 2001;345:810-816.

2. Jacob LR. Prescription to over-the-counter drug reclassification. Am Fam Physician. 1998;57:2209-2214.

3. Snyder K. Marketing consumer trends driving switch-hitters up: prescription drugs changing to over-the-counter status. Drug Topics. 1997;89:141

4. Snyder K. Rising tide: new wave of Rx-to-OTC switches coming. Drug Topics. 1995;207:47-49.

5. Rosenau PV. Rx to OTC switch movement. Med Care Rev. 1994;51: 429-267.

6. Pyridium tablets. In: Sifton DW, Murray L. Physician's Desk Reference. 54th ed. New Jersey. Medical Economics; 2000:3164.

7. Stuart B, Grana J. Ability to pay and the decision to medicate. Med Care. 1998;36:202-211.

8. Aday LA, Anderson R. A framework for the study of access to medical care. Health Serv Res. 1974;9:208-220.

9. Sommers SD, Chaiyakunapruk N, Gardner JS, Winkler J. The emergency contraception collaborative prescribing experience in Washington State. J of Am Pharm Assoc. 2001;41:60-66

10. Botstein P. Criteria for switching new molecular entities form prescription to over-the-counter. Drug Inf J. 1990;24:29-31.

11. Rubin Rita. Heartburn sufferers could get OTC Prilosec relief. Available at http://www.usatoday.com/news/health/2002-05-30prilosec.htm. Accessed February 2003.

12. Kaledin Elizabeth. FDA mulls over-the-counter measures. Available at http://www.cbsnews.com/stories/2000/06/29/national/ main210949.shtml. Accessed February 2003 